\title{
Dynamic Analysis of a Plankton-Herbivore State- Dependent Impulsive Model With Action Threshold Depending On The Density and Its Changing Rate
}

Wei Li

Shandong University of Science and Technology

Tonghua Zhang

Swinburne University of Technology - Hawthorn Campus: Swinburne University of Technology

\section{Yufei Wang}

Shandong University of Science and Technology

Huidong Cheng ( $\square$ chd900517@sdust.edu.cn )

Shandong University of Science and Technology

\section{Research Article}

Keywords: State-dependent impulsive model, action threshold, Poincaré map, periodic solution.

Posted Date: July 21st, 2021

DOI: https://doi.org/10.21203/rs.3.rs-710111/v1

License: (9) This work is licensed under a Creative Commons Attribution 4.0 International License.

Read Full License

Version of Record: A version of this preprint was published at Nonlinear Dynamics on November 10th, 2021. See the published version at https://doi.org/10.1007/s11071-021-07022-w. 


\title{
Dynamic analysis of a plankton-herbivore state-dependent impulsive model with action threshold depending on the density and its changing rate
}

\author{
Wei $\mathrm{Li}^{1}$, Tonghua Zhang ${ }^{2}$, Yufei Wang ${ }^{1}$, Huidong Cheng ${ }^{1 d *}$ \\ 1.College of Mathematics and System Sciences, Shandong University of Science and Technology. \\ Qingdao 266590, Shandong, People's Republic of China \\ 2. Department of Mathematics, Swinburne University of Technology, Hawthorn 3122 VIC Australia \\ Email: tonghuazhang@swin.edu.au
}

\begin{abstract}
A plankton-herbivore state-dependent impulsive model with nonlinear impulsive functions and action threshold including population density and rate of change is proposed. Since the use of action threshold makes the model have complex phase set and pulse set, we adopt the Poincaré map as a tool to study its complex dynamics. The Poincaré map is defined on the phase set and its properties in different situations are analyzed. Furthermore, the periodic solution of model are discussed, including the existence and stability conditions of the order-1 periodic solution and the existence of the order-k $(k \geq 2)$ periodic solutions. Compared with the fixed threshold in the existing literature, our results show that the use of action threshold is more practical, which is conducive to the sustainable development of population and makes people obtain more economic benefits. The analysis method used in this paper can study the complex dynamics of the model more comprehensively and deeply.
\end{abstract}

Keywords: State-dependent impulsive model, action threshold, Poincaré map, periodic solution.

\section{Introduction}

Plankton are at the bottom of the marine food chain. They are widely distributed and highly reproductive, forming the foundation of the marine food web. In recent years, human activities have seriously interfered with the ecosystem, and over exploitation of marine organisms has become a common problem. To better understand these interactions, more and more scholars pay attentions to the plankton-related models, for example, a plankton-fish model under stochastic fluctuations was proposed and studied in [1], a phytoplankton-zooplankton model with harvesting was proposed in [2], the interactions between plankton and other species and effects of environmental changes were studied in [3], a random nutrition- plankton food chain model with nutritional

\footnotetext{
${ }^{*}$ Corresponding author. Email: chd900517@sdust.edu.cn(Huidong Cheng)
} 
recovery was proposed by [4], a stochastic model of phytoplankton-zooplankton in impulsive pollution environment was proposed by [5], in references [6-8] researchers investigated the pattern dynamics.

For a herbivore-plankton model with cannibalism, the existence and stability of order-1 periodic solution were discussed in [9], where the model was a system of Impulsive Differential Equations (IDEs), which has more complex and abundant dynamics, but can fully take into account the impact of instantaneous mutations on the state and more deeply reflect the law of things changing. IDEs are widely used in biology, medicine, control theory and other fields [10-13]. In biology, the impulsive differential equation can be proposed to incorporate the possible changes in the population into the research model, more information can be found from [14,15]. In biology, the statedependent feedback control means that the control will be implemented only when the population reaches a given threshold level [16-18]. This threshold control strategy is applied to solve many practical biological problems, such as the influence between biological populations [19-21], integrated pest management [22-24], infectious disease control [25-27], fishery harvesting [4,5,28-30], etc. Therefore, it is of great practical significance to establish the corresponding mathematical model to describe and study the state-dependent feedback control strategy. The impulsive semidynamical system or the state-dependent impulsive differential equation can characterize threshold control strategies very naturally, and a comprehensive analysis of the equations can reveal many important biological conclusions.

In controlling actual biological populations, we focus on when to take control and how to control them. The state-dependent feedback control as mentioned above is carried out on the biological population density when it reaches a fixed threshold in [10,31]. Thus, the impulsive set is a straight line, which makes the calculation relatively simple. But from the perspective of biology, the fixed threshold cannot be combined with the actual situation of the biological population to determine when to control the biological population. There are two cases in practice: (a) the population density is small but the the rate of change is high, which often occurs in the early stage of population growth; (b) the population density is large, but the rate of change is small. Therefore, the threshold of the model needs to contains both population density and change rate, i.e. the proportionally dependent action threshold. In the process of releasing and fishing the biological population, different factors need to be integrated to make the control methods adopted more in line with the development law of biological populations. Therefore, the adopted state-dependent impulsive model needs to consider both the selection of threshold and impulsive function.

In [9] authors only performed a pulse control when the fixed threshold is reached, and the impulsive function used is relatively simple. The more complex dynamic properties of the model are also not studied due to the difficulties in analysis. Now based on the work of [9] and our above analysis, we will propose a plankton-herbivore state-dependent impulsive model with action threshold and nonlinear impulsive function:

$$
\left\{\begin{array}{l}
\frac{d p(t)}{d t}=p(t)\left(k-p(t)-\frac{h(t)}{1+p(t)}\right), \\
\frac{d h(t)}{d t}=h(t)\left(\frac{p(t)}{1+p_{\tau}(t)}-a h(t)\right), \\
p\left(t^{+}\right)=p(t)+\frac{\sigma h^{2}(t)}{1+\eta p(t)}, \\
h\left(t^{+}\right)=h(t)-\frac{\sigma h_{1} p+v_{1} \frac{d p}{d t}>W,}{h(t)+\mu},
\end{array}\right\} \quad u_{1} p+v_{1} \frac{d p}{d t}=W,
$$

where $p$ and $h$ represent the plankton and herbivore density, respectively, $k$ denotes the biological 
carrying capacity and $a$ indicates the density-dependent influence of herbivores. The parameters $u_{1}, v_{1}$ and $W$ are positive values and $u_{1}+v_{1}=1$. When the amount of plankton reaches the action threshold $u_{1} p+v_{1} \frac{d p}{d t}=W$, actions will be taken to control the plankton and herbivore populations. The nonlinear impulsive functions related to the density and fishing rate of the biological population are adopted as follows:

$$
\alpha=\frac{\tau}{1+\eta p(t)} \text { and } \beta=-\frac{\sigma h^{2}(t)}{h(t)+\mu},
$$

where $\tau$ is the maximum number of plankton released, $\eta$ denotes the morphological parameter. $\sigma$ indicates the maximum catch rate of herbivores and $\mu$ represents its half-saturation constant. After impulse control, the number of herbivores and plankton changes to $h(t)-\frac{\sigma h^{2}(t)}{h(t)+\mu}$ and $p(t)+\frac{\tau}{1+\eta p(t)}$, respectively. The use of the action threshold makes the pulse set and the phase set become two curves, and the nonlinear pulse function complicates the model. Further, the dynamic properties of model become very complicated. The global dynamics of the state-dependent impulsive model at this time deserve our in-depth and comprehensive study.

From [9] or straightforward analysis, we obtain that when the system (1) has no impulsive effect it becomes

$$
\left\{\begin{array}{l}
\frac{d p(t)}{d t}=p(t)\left(k-p(t)-\frac{h(t)}{1+p(t)}\right), \\
\frac{d h(t)}{d t}=h(t)\left(\frac{p(t)}{1+p(t)}-a h(t)\right),
\end{array}\right.
$$

which has nullclines:

$$
L_{1}: h=(k-p)(1+p), L_{2}: h=\frac{p}{a(1+p)},
$$

and $O(0,0)$ is the boundary equilibrium point, which is unstable and $E_{0}(k, 0)$ is a saddle point. The system (2) has an internal equilibrium point $E^{*}\left(p^{*}, h^{*}\right)$, where $p^{*}=a\left(k-p^{*}\right)\left(1+p^{*}\right)^{2}, h^{*}=\frac{p^{*}}{a\left(1+p^{*}\right)}$, and when $k<2 p^{*}+2$, the $E^{*}$ is stable; when $k>2 p^{*}+2, E^{*}$ is unstable, and the occurrence of the a Hopf bifurcation makes a stable limit cycle exist when $k=2 p^{*}+2$.

In our study here, we consider the case where $E^{*}$ is stable. Thus, in the rest of discuss we assume that $k<2 p^{*}+2$.

\section{Definition of the Poincaré map}

For biologically meaningful, we restrict our discussion to

$$
R_{+}^{2}=\{(p, h): p \geq 0, h \geq 0\} .
$$

Since system (1) uses an action threshold that includes both population density and rate of change, the impulsive set and phase set are two curves. According to $u_{1} p+v_{1} \frac{d p}{d t}=W$ and the first equation of system (1), we get

$$
h=\left(k-p-\frac{W-u_{1} p}{v_{1} p}\right)(1+p)
$$

denoted by $L_{M}$. From $p^{+}=p+\frac{\tau}{1+\eta p}$, we obtain

$$
p=\frac{\eta p^{+}-1+\sqrt{\eta^{2}\left(p^{+}\right)^{2}+2 \eta p^{+}-4 \eta \tau+1}}{2 \eta} .
$$


Therefore,

$$
\begin{aligned}
& h=\left(k-p-\frac{W-u_{1} p}{v_{1} p}\right)(1+p)=\left(k-\frac{\eta p^{+}-1+\sqrt{\eta^{2}\left(p^{+}\right)^{2}+2 \eta p^{+}-4 \eta \tau+1}}{2 \eta}\right. \\
& \left.-\frac{W-u_{1} \frac{\eta p^{+}-1+\sqrt{\eta^{2}\left(p^{+}\right)^{2}+2 \eta p^{+}-4 \eta \tau+1}}{2 \eta}}{v_{1} \frac{\eta p^{+}-1+\sqrt{\eta^{2}\left(p^{+}\right)^{2}+2 \eta p^{+}-4 \eta \tau+1}}{2 \eta}}\right)\left(1+\frac{\eta p^{+}-1+\sqrt{\eta^{2}\left(p^{+}\right)^{2}+2 \eta p^{+}-4 \eta \tau+1}}{2 \eta}\right) .
\end{aligned}
$$

Let

$$
h^{+}=h\left(1-\frac{\sigma h}{h+\mu}\right)=\phi\left(p^{+}\right)
$$

denoted by $L_{N}$. In what follows, unless special instructions, the initial point $S_{0}^{+}\left(p_{0}^{+}, h_{0}^{+}\right)$is on the curve $L_{N}$. Since the trajectory starting from $L_{N}$ may not reach $L_{M}$ depending on the initial values, the specific ranges of the impulsive set and the phase set can be obtained according to different positional relations between the trajectory and the equilibrium point (see Figure 1).

\subsection{Case $\left(C_{1}\right): p^{*} \leq p_{M}^{*} \cdot$}

Let $p_{M}^{*}$ be the horizontal coordinate of curve $L_{M}$ under $h=h^{*}=\frac{p^{*}}{a\left(1+p^{*}\right)}$. Since $p^{*} \leq p_{M}^{*}$, there must be a point $T_{1}\left(p_{T_{1}}, h_{T_{1}}\right)$ on $L_{N}$ such that $\Gamma_{T_{1}}$ and $L_{N}$ are tangent. And the trajectory $\Gamma_{T_{1}}$ intersects with the $L_{M}$ at point $T_{2}\left(p_{T_{2}}, h_{T_{2}}\right)$ (Figure 1(a)). Thus, the trajectory of the starting point from the phase set must reach the impulsive set at some time. Therefore, in this case, the range of the impulsive set and phase set is as follows:

$$
M_{1}=\left\{(p, h) \mid p \geq p_{T_{2}}, h \geq h_{T_{2}}\right\}
$$

and

$$
N_{1}=\left\{\left(p^{+}, h^{+}\right) \mid p^{+} \geq p_{T_{2}}+\frac{\tau}{1+\eta p_{T_{2}}}, h^{+} \geq h_{T_{2}}-\frac{\sigma h_{T_{2}}^{2}}{h_{T_{2}}+\mu}\right\},
$$

respectively, where $(p, h)$ is a point on the curve $L_{M}: h=\left(k-p-\frac{W-u_{1} p}{v_{1} p}\right)(1+p)$ and $\left(p^{+}, h^{+}\right)$is a point on the curve $L_{N}$.

\subsection{Case $\left(C_{2}\right): p^{*}>p_{M}^{*}$.}

In this case, since $p^{*}>p_{M}^{*}$, there must exist a point $A\left(p_{A}, h_{A}\right)$ on impulsive set, which makes the trajectory $\Gamma_{A}$ tangent to $L_{M}$. Thus, the trajectory $\Gamma_{A}^{-}$and $L_{1}$ intersect at point $E_{1}\left(p_{E_{1}}, h_{E_{1}}\right)$. Let $p_{N_{1}}$ be the horizontal coordinate of curve $L_{N}$ under $h=h_{E_{1}}$. We have the following two different situations:

(I) $p_{E_{1}}<p_{N_{1}}$.

If $p_{E_{1}}<p_{N_{1}}$, the situation is similar to the Case $\left(C_{1}\right)$. At this time $\Gamma_{A}$ and $L_{N}$ have no intersection and all points starting from the phase set will eventually hit the impulsive set. There is a point $B_{1}\left(p_{B_{1}}, h_{B_{1}}\right)$ on $L_{N}$ such that the trajectory $\Gamma_{B_{1}}$ is tangent to $L_{N}$. And $\Gamma_{B_{1}}$ reaches the impulsive set at point $B_{2}\left(p_{B_{2}}, h_{B_{2}}\right)$ (Figure $1(\mathrm{~b})$ ). Therefore, the range of the impulsive set is:

$$
M_{2}=\left\{(p, h) \mid p \geq p_{B_{2}}, h \geq h_{B_{2}}\right\},
$$




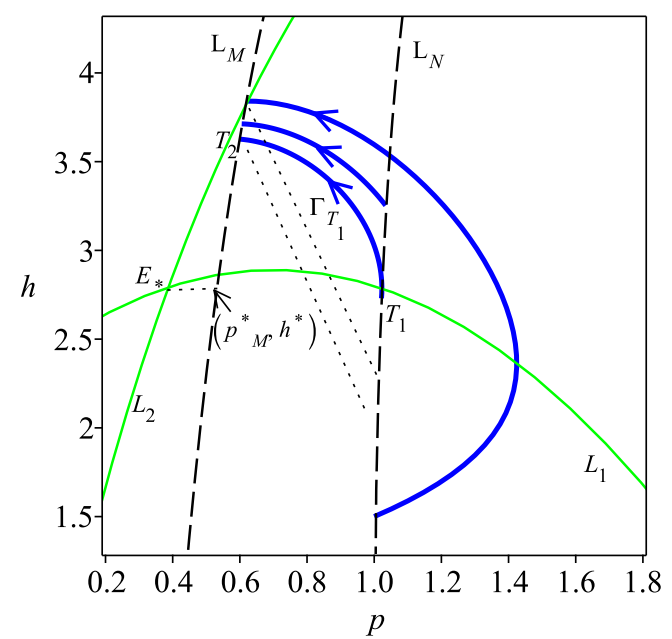

(a)

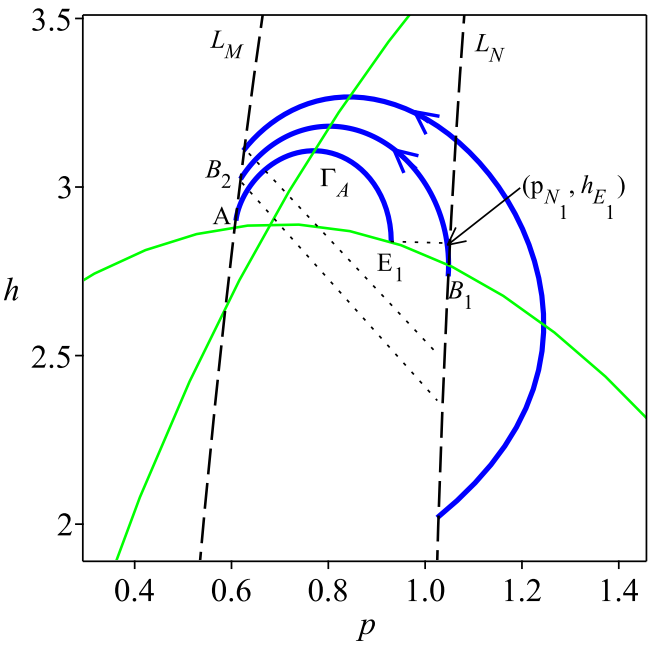

(b)

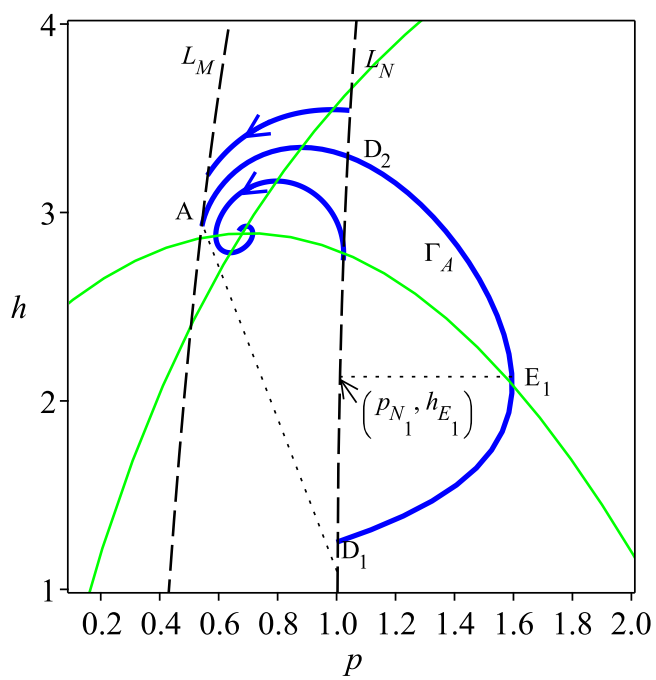

(c)

Figure 1: The trajectories of system (1) under different cases, where the parameter values are $k=2.4, \eta=5, \tau=1.8, \mu=0.4, \sigma=0.8$. (a): $W=0.43, a=0.1,(\mathrm{~b}): W=0.48, a=0.14$, and (c): $W=0.43, a=0.14$.

the corresponding phase set is

$$
N_{2}=\left\{\left(p^{+}, h^{+}\right) \mid p^{+} \geq p_{B_{2}}+\frac{\tau}{1+\eta p_{B_{2}}}, h^{+} \geq h_{B_{2}}-\frac{\sigma h_{B_{2}}^{2}}{h_{B_{2}}+\mu}\right\} .
$$

(II) $p_{E_{1}}>p_{N_{1}}$.

If $p_{E_{1}}>p_{N_{1}}$, the curve $\Gamma_{A}^{-}$intersects the line $L_{N}$ at points $D_{1}\left(p_{D_{1}}, h_{D_{1}}\right)$ and $D_{2}\left(p_{D_{2}}, h_{D_{2}}\right)$, where $h_{D_{1}}<h_{D_{2}}$. There is no intersection between the system trajectory and the impulsive set whose initial point is between $D_{1}$ and $D_{2}$ on $L_{N}$ at this case (Figure 1(c)). So we give the following range of impulsive set:

$$
M_{3}=\left\{(p, h) \mid p \geq p_{A}, h \geq h_{A}\right\}
$$


and the range of the phase set is

$$
N_{3}=\left\{\left(p^{+}, h^{+}\right) \mid p^{+} \in\left[p_{A}+\frac{\tau}{1+\eta p_{A}}, p_{D_{1}}\right] \cup\left[p_{D_{2}},+\infty\right), h^{+} \in\left[h_{A}-\frac{\sigma h_{A}^{2}}{h_{A}+\mu}, h_{D_{1}}\right] \cup\left[h_{D_{2}},+\infty\right)\right\} .
$$

According to the above discussion, the Poincaré map is constructed. Given an initial point $S_{0}^{+}\left(p_{0}^{+}, h_{0}^{+}\right) \in N$, the trajectory starting from $S_{0}^{+}$can be expressed as

$$
\Gamma\left(t, t_{0}, S_{0}^{+}\right)=\Gamma\left(p\left(t, t_{0},\left(p_{0}^{+}, h_{0}^{+}\right)\right), h\left(t, t_{0},\left(p_{0}^{+}, h_{0}^{+}\right)\right)\right) .
$$

For $\forall S_{i}^{+}\left(p_{i}^{+}, h_{i}^{+}\right) \in N$, the trajectory from $S_{i}^{+}$reaches the impulsive set at point $S_{i+1}\left(p_{i+1}, h_{i+1}\right)$ elapsed time $\tilde{t}$. We express this process as

$$
\Gamma\left(\tilde{t}, p_{i}^{+}, h_{i}^{+}\right)=\Gamma\left(\tilde{t}, p_{i+1}, h_{i+1}\right)=\Gamma\left(p^{+}\left(\tilde{t}, p_{i}^{+}, h_{i}^{+}\right), h^{+}\left(\tilde{t}, p_{i}^{+}, h_{i}^{+}\right)\right)=\Gamma\left(p_{i+1}, h_{i+1}\right),
$$

where $h_{i+1}=\left(k-p_{i+1}-\frac{w-u_{1} p_{i+1}}{v_{1} p_{i+1}}\right)\left(1+p_{i+1}\right)$ and $h_{i+1}=h^{+}\left(\tilde{t}, p_{i}^{+}, h_{i}^{+}\right)$. According to the CauchyLipschitz Theorem, $h_{i+1}$ is only expressed by $h_{i}^{+}$. Define $h_{i+1}=\xi\left(h_{i}^{+}\right)$. Then the expression of Poincaré map is

$$
h_{i+1}^{+}=h_{i+1}-\frac{\sigma h_{i+1}^{2}}{h_{i+1}+\mu}=\xi\left(h_{i}^{+}\right)-\frac{\sigma \xi\left(h_{i}^{+}\right)^{2}}{\xi\left(h_{i}^{+}\right)+\mu}=Q_{M}\left(h_{i}^{+}\right) .
$$

Consider the scalar differential equation from system (2):

$$
\left\{\begin{array}{l}
\frac{d h}{d p}=\frac{h\left(\frac{p}{1+p}-a h\right)}{p\left(k-p-\frac{h}{1+p}\right)} \triangleq g(p, h), \\
h(W)=h_{0}^{+} .
\end{array}\right.
$$

The function $g(p, h)$ is continuously differentiable. Let $p_{0}^{+}=X, h_{0}^{+}=Z$, and $S_{0}^{+}\left(p_{0}^{+}, h_{0}^{+}\right) \in N$. Now define

$$
h(p)=h(p ; X, Z)=h(p, Z),
$$

where the value of $p$ is between the phase set and the impulsive set. Then it follows from (6) that

$$
h(p, Z)=Z+\int_{p}^{X} g(z, h(z, Z)) d z .
$$

From equations (5) and (8), Poincaré map $Q(Z)$ can be expressed as

$$
Q(Z)=h(X, Z)-\frac{\sigma h^{2}(X, Z)}{h(X, Z)+\mu}
$$

\section{The natures of Poincaré map}

We now explore the nature of the Poincaré map $Q(Z)$, which are summarised in the following theorem. The details are omitted here, and the proof of the theorem can be found in article [32].

Theorem 3.1. Assume $k<2 p^{*}+2$ and $p^{*} \leq p_{M}^{*}$. The main properties of the Poincaré map are listed(Figure 2): 


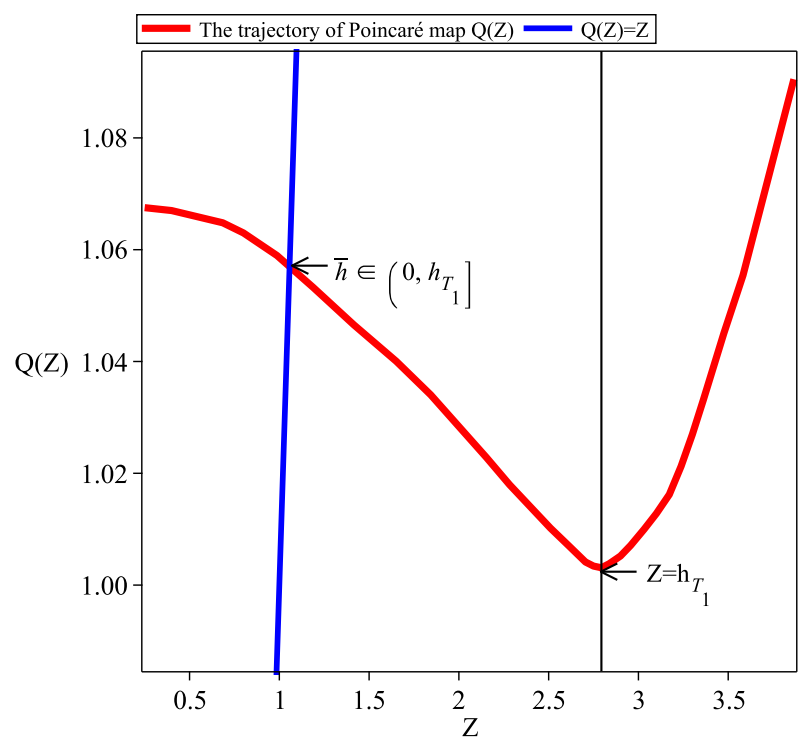

(a)

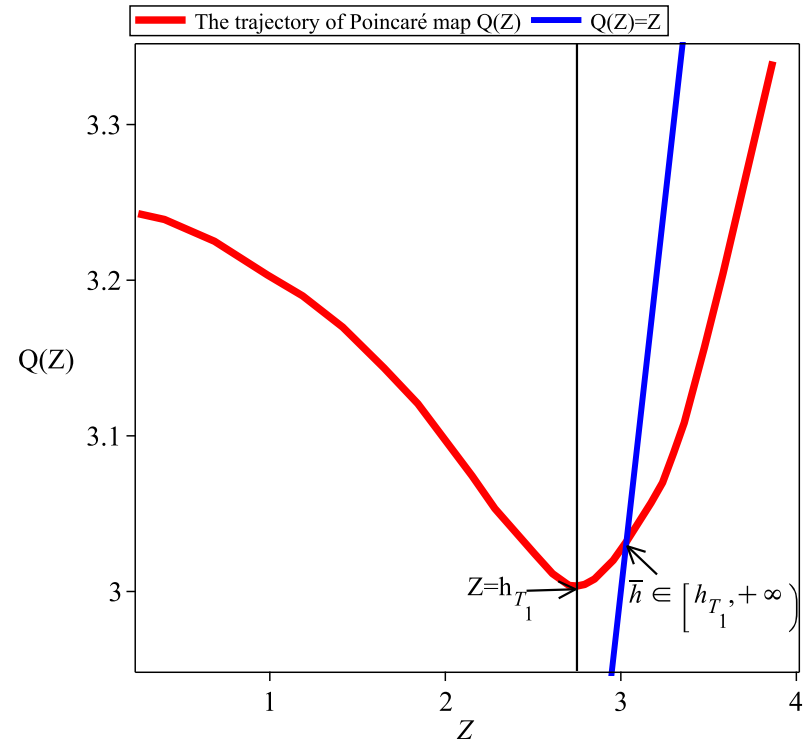

(b)

Figure 2: The Poincaré map $Q(Z)$ and fixed point in case $\left(C_{1}\right)$, where the parameter values are $k=2.4, a=0.1, W=0.43, \eta=5, \tau=1.8, u_{1}=0.9, v_{1}=0.1(a) \mu=0.4, \sigma=0.8(b) \mu=0.8, \sigma=0.2$.

(i) $Q(Z)$ is defined on $(0,+\infty)$, and it is monotonically decreasing on $\left(0, h_{T_{1}}\right]$ and monotonically increasing on $\left[h_{T_{1}},+\infty\right)$. Furthermore, $Q(Z)$ reaches its minimum value at $h_{T_{1}}$, and no maximum value.

(ii) $Q(Z)$ is continuously differentiable in $(0,+\infty)$, and admits a unique fixed point.

When $p^{*}>p_{M}^{*}$ and $p_{E_{1}}<p_{N_{1}}$, the natures of Poincaré map is similar to Case $\left(C_{1}\right)$. Therefore next we discuss the situation where $p_{E_{1}}>p_{N_{1}}$.

Theorem 3.2. Assume $k<2 p^{*}+2, p^{*}>p_{M}^{*}$ and $p_{E_{1}}>p_{N_{1}}$. The main properties of the Poincaré map are listed(Figure 3):

(i) $Q(Z)$ is defined on $\left(0, h_{D_{1}}\right] \cup\left[h_{D_{2}},+\infty\right)$. On $\left(0, h_{D_{1}}\right]$ the map $Q(Z)$ decreases and on $\left[h_{D_{2}},+\infty\right)$ the map $Q(Z)$ increases.

(ii) $Q(Z)$ is continuously differentiable in its domain.

(iii) If $Q\left(h_{D_{1}}\right)<h_{D_{1}}, Q(Z)$ has a unique fixed point on $\left(0, h_{D_{1}}\right]$. If $Q\left(h_{D_{2}}\right)>h_{D_{2}}, Q(Z)$ has no fixed point.

Proof. (i) When $p_{E_{1}}>p_{N_{1}}$, we know the trajectory $\Gamma_{A}$ and the phase set $L_{N}$ intersect at two points $D_{1}$ and $D_{2}$, and $\Gamma_{A}$ is tangent to the phase set $L_{M}$. For $\forall S_{i}^{+}\left(p_{i}^{+}, h_{i}^{+}\right) \in N$, if $h_{i}^{+} \in\left(h_{D_{1}}, h_{D_{2}}\right)$, the trajectory starting from the phase set cannot reach the impulsive set. Therefore, $Q(Z)$ is well defined when $Z \in\left(0, h_{D_{1}}\right] \cup\left[h_{D_{2}},+\infty\right)$. So Poincaré map is meaningful in the interval $\left(0, h_{D_{1}}\right] \cup\left[h_{D_{2}},+\infty\right)$.

Suppose there are two points $S_{k_{1}}^{+}\left(p_{k_{1}}^{+}, h_{k_{1}}^{+}\right)$and $S_{k_{2}}^{+}\left(p_{k_{2}}^{+}, h_{k_{2}}^{+}\right)$on the phase set. If $h_{k_{1}}^{+}, h_{k_{2}}^{+} \in$ $\left[h_{D_{2}},+\infty\right)$, let $h_{k_{1}}^{+}<h_{k_{2}}^{+}$. From the trajectory of system (1) we get through time $\tilde{t}, \Gamma\left(\tilde{t}, p_{i}^{+}, h_{i}^{+}\right)=$ $\Gamma\left(\tilde{t}, p_{i+1}, h_{i+1}\right)$. Since the uniqueness of the solution we have $h_{k_{1}+1}<h_{k_{2}+1}$. From the expression of Poincaré map $Q(Z)$, we get $Q\left(h_{k_{1}+1}\right)<Q\left(h_{k_{2}+1}\right)$. So $Q(Z)$ is monotonically increasing on $\left[h_{D_{2}},+\infty\right)$. 


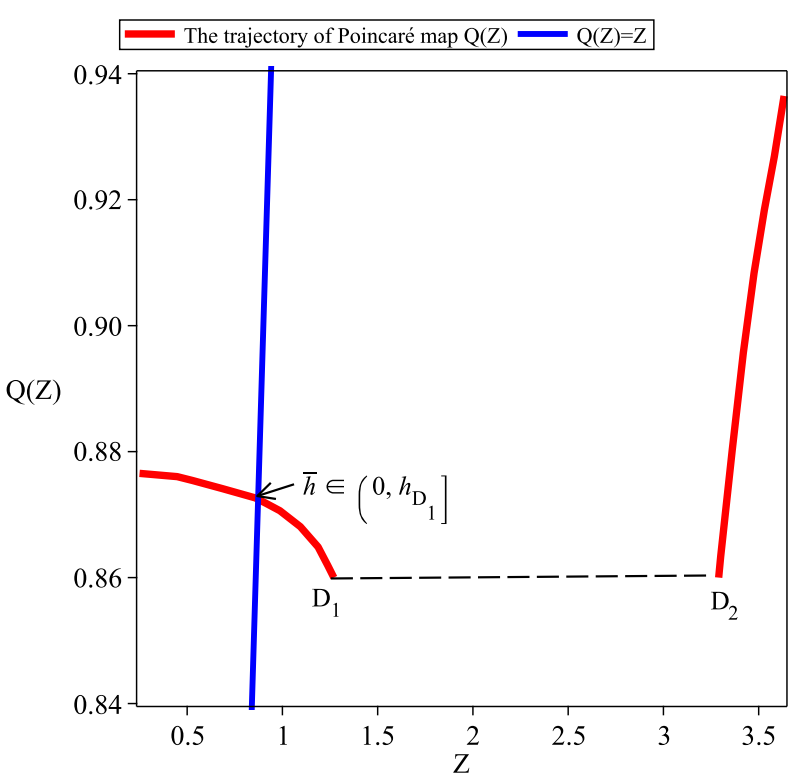

(a)

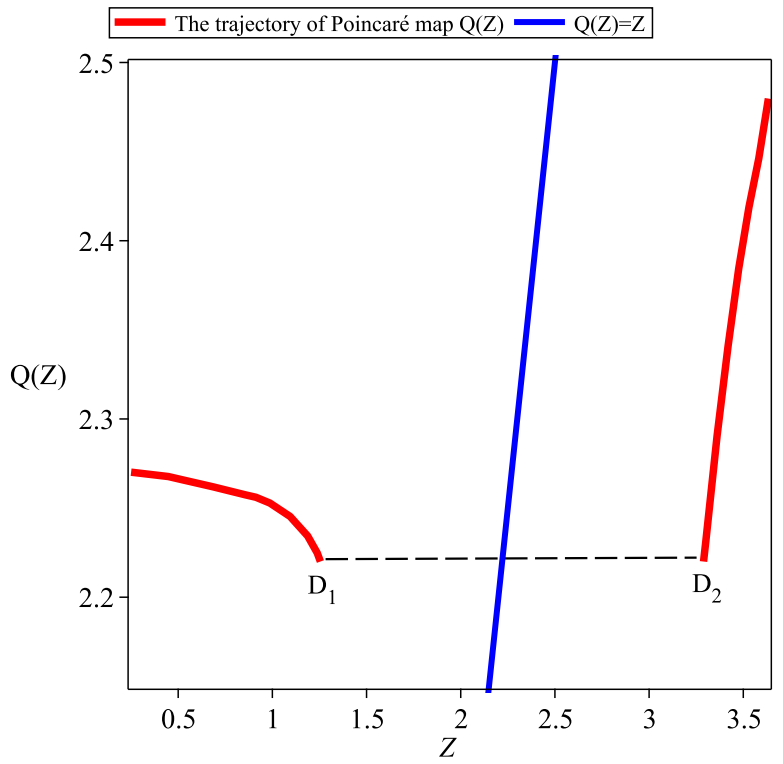

(b)

Figure 3: The Poincaré map $Q(Z)$ and its fixed point in case $\left(C_{2}\right)(\mathrm{II})$, where the parameter values are $k=2.4, a=0.14, W=0.43, \eta=5, \tau=1.8, u_{1}=0.9, v_{1}=0.1(a) \mu=0.4, \sigma=0.8(b) \mu=$ $0.8, \sigma=0.3$.

When $h_{k_{1}}^{+}, h_{k_{2}}^{+} \in\left(0, h_{D_{1}}\right]$ and $h_{k_{1}}^{+}<h_{k_{2}}^{+}$. The trajectory starts from point $S_{k_{1}}^{+}, S_{k_{2}}^{+}$through the isometric line $L_{1}$ and intersects the $L_{N}$ at the point $S_{k_{1}}^{\prime}\left(p_{k_{1}}^{\prime}, h_{k_{1}}^{\prime}\right)$ and $S_{k_{2}}^{\prime}\left(p_{k_{2}}^{\prime}, h_{k_{2}}^{\prime}\right)$. It is easy to get that $h_{k_{1}}^{\prime}>h_{k_{2}}^{\prime}$. Then $Q_{M}\left(h_{k_{1}}^{\prime}\right)>Q_{M}\left(h_{k_{2}}^{\prime}\right)$ from the uniqueness of the solution. So $Q(Z)$ is monotonically decreasing on $\left(0, h_{D_{1}}\right]$.

(ii) According to the equation (6), we obtain that the function $g(p, h)$ is continuously differentiable. Therefore from the continuous differentiability theorem of differential equations, Poincaré map $Q(Z)$ is continuously differentiable on $\left(0, h_{D_{1}}\right] \cup\left[h_{D_{2}},+\infty\right)$ in the first quadrant.

(iii) The curve $\Gamma_{A}$ from $D_{1}$ reaches the impulsive set at point $A$, and then arrives in phase set at point $A^{+}\left(p_{A}^{+}, h_{A}^{+}\right)$. If $Q\left(h_{D_{1}}\right)<h_{D_{1}}$, then $h_{A}^{+}=Q\left(h_{D_{1}}\right) \in\left(0, h_{D_{1}}\right]$ and $h_{A}^{+}<h_{D_{1}}$. Since $Q(Z)$ is monotonically decreases on $\left(0, h_{D_{1}}\right]$, then $Q\left(h_{A}^{+}\right)>Q\left(h_{D_{1}}\right)=h_{A}^{+}$. We get there exists $\bar{h} \in\left(h_{A}^{+}, h_{D_{1}}\right)$ such that $Q(\bar{h})=\bar{h}$ from the zero point existence theorem and the continuous differentiability of $Q(Z)$. Therefore $Q(Z)$ has a unique fixed point on $\left(0, h_{D_{1}}\right]$.

If $Q\left(h_{D_{2}}\right)>h_{D_{2}}$, for any $h_{i} \in\left[h_{D_{2}},+\infty\right)$, the trajectory from point $S_{i}\left(p_{i}, h_{i}\right)$ reaches the $L_{M}$ at point $S_{i+1}\left(p_{i+1}, h_{i+1}\right)$. Then $S_{i}^{+}\left(p_{i}^{+}, h_{i}^{+}\right)$is pulsed to $S_{i+1}^{\prime}\left(p_{i+1}^{\prime}, h_{i+1}^{\prime}\right)$. According to the trajectory of system (1), we easily obtain that $h_{i}<h_{i+1}<h_{i+1}^{\prime}$. Therefore there is no $\bar{h}_{1} \in\left[h_{D_{2}},+\infty\right)$ such that $Q\left(\bar{h}_{1}\right)=\bar{h}_{1}$.

\section{Order-k periodic solution}

From the previous section we know that the system has a unique fixed point, which indicates that the model(1) has a unique order-1 periodic solution.

Theorem 4.1. In case $\left(C_{1}\right)$, system(1) has a unique order-1 periodic solution. Furthermore, we obtain that

(i) If $Q\left(h_{T_{1}}\right)>h_{T_{1}}$, the order-1 periodic solution is globally stable; 
(ii) If $Q\left(h_{T_{1}}\right)<h_{T_{1}}$, the order-1 periodic solution is globally stable when $Q^{2}\left(h_{0}^{+}\right)<h_{0}^{+}$for any $h_{0}^{+} \in\left[\bar{h}, h_{T_{1}}\right]$.

Proof. From (6), we get $h_{i+1}^{+}=\xi\left(h_{i}^{+}\right)-\frac{\sigma \xi\left(h_{i}^{+}\right)^{2}}{\xi\left(h_{i}^{+}\right)+\mu}=Q\left(h_{i}^{+}\right)$. Therefore, the trajectory with $S_{0}^{+}\left(p_{0}^{+}, h_{0}^{+}\right) \in$ $N$ as the initial point intersects the impulsive set at the point $S_{1}\left(p_{1}, h_{1}\right)$, and will arrive at point $S_{1}^{+}\left(p_{1}^{+}, h_{1}^{+}\right) \in N$ through the action of the impulse. We express this process as $Q\left(h_{0}^{+}\right)=$ $\xi\left(h_{0}^{+}\right)-\frac{\sigma \xi\left(h_{0}^{+}\right)^{2}}{\xi\left(h_{0}^{+}\right)+\mu}=h_{1}^{+}$. Through the similar process we have $Q\left[Q\left(h_{0}^{+}\right)\right]=Q\left(h_{1}^{+}\right)=h_{2}^{+}=Q^{2}\left(h_{0}^{+}\right)$. Further we get $Q^{k}\left(h_{0}^{+}\right)=h_{k}^{+}$.

(i) In case $\left(C_{1}\right)$, from Theorem 3.1 we get if $Q\left(h_{T_{1}}\right)>h_{T_{1}}$, the Poincaré map $Q(Z)$ exists unique fixed point $\bar{h} \in\left[h_{T_{1}},+\infty\right)$. So we have the following two situations:

If $h_{0}^{+} \in\left(h_{T_{1}}, \bar{h}\right], Q(Z)$ monotonically increasing in this interval. Therefore

$$
\bar{h}=Q(\bar{h})>Q\left(h_{0}^{+}\right)>Q\left(h_{T_{1}}\right)>h_{T_{1}}
$$

further through the trajectory and the nature of $Q(Z)$ we get

$$
\bar{h}=Q(\bar{h})>Q\left(h_{1}^{+}\right)>Q\left(h_{0}^{+}\right) .
$$

Therefore

$$
\bar{h}>\ldots>Q^{k}\left(h_{0}^{+}\right)>Q^{k-1}\left(h_{0}^{+}\right)>\ldots>Q\left(h_{0}^{+}\right)>h_{T_{1}},
$$

which represents that $Q^{k}\left(h_{0}^{+}\right)$increases monotonically and $\lim _{n \rightarrow+\infty} Q^{k}\left(h_{0}^{+}\right)=\bar{h}$.

If $h_{0}^{+} \in(\bar{h},+\infty)$, through similar analysis we obtain that

$$
h_{0}^{+}>Q\left(h_{0}^{+}\right)>Q(\bar{h})=\bar{h}, Q\left(h_{0}^{+}\right)>Q\left(h_{1}^{+}\right)>Q(\bar{h})=\bar{h},
$$

Further we have

$$
h_{0}^{+}>Q\left(h_{0}^{+}\right) \ldots>Q^{k-1}\left(h_{0}^{+}\right)>Q^{k}\left(h_{0}^{+}\right)>\ldots>\bar{h} .
$$

Therefore, $Q^{k}\left(h_{0}^{+}\right)$decreases monotonically and $\lim _{n \rightarrow+\infty} Q^{k}\left(h_{0}^{+}\right)=\bar{h}$. The order-1 periodic solution is globally stable if $Q\left(h_{T_{1}}\right)>h_{T_{1}}$.

(ii) Sufficient condition: We know when $Q\left(h_{T_{1}}\right)<h_{T_{1}}, Q(Z)$ has unique fixed point $\bar{h} \in\left(0, h_{T_{1}}\right]$ from Theorem 3.1. And $Q(Z)$ monotonically decreasing on $\left(0, h_{T_{1}}\right]$. Therefore if $Q^{2}\left(h_{0}^{+}\right)<h_{0}^{+}$for any $h_{0}^{+} \in\left[\bar{h}, h_{T_{1}}\right]$, then

$$
h_{T_{1}}>h_{0}^{+}>Q^{2}\left(h_{0}^{+}\right)>\bar{h}>Q\left(h_{0}^{+}\right)>Q\left(h_{T_{1}}\right) .
$$

Further we derive that

$$
h_{T_{1}}>Q^{2 k}\left(h_{0}^{+}\right)>\bar{h}>Q^{2 k+1}\left(h_{0}^{+}\right)>Q\left(h_{T_{1}}\right) .
$$

Therefore we have $\lim _{k \rightarrow \infty} Q^{2 k}\left(h_{0}^{+}\right)=\lim _{k \rightarrow \infty} Q^{2 k+1}\left(h_{0}^{+}\right)=\bar{h}$ from monotone bounded theorem. Then the order-1 periodic solution is globally stable.

Necessary condition: When the order-1 periodic solution $\bar{h}$ is globally stable, we assume there exists $\tilde{h}_{1}^{+} \in\left(\bar{h}, h_{T_{1}}\right]$ such that $Q^{2}\left(\tilde{h}_{1}^{+}\right) \geq \tilde{h}_{1}^{+}$. Then there exists a $\tilde{h}_{2}^{+} \in(\bar{h}-\varepsilon, \bar{h}-\varepsilon)$ and $Q^{2}\left(\tilde{h}_{2}^{+}\right)<\tilde{h}_{2}^{+}$ from the stability of $\bar{h}$, where $\varepsilon$ is a small enough number. Since Poincaré map is continuous, then there is at least a $\bar{h}^{\prime}$ between $\tilde{h}_{1}^{+}$and $\tilde{h}_{2}^{+}$which makes $Q^{2}\left(\bar{h}^{\prime}\right)=\bar{h}^{\prime}$. At this time, system (1) has order-2 periodic solution which is inconsistent with the known conditions. Therefore, if the order-1 periodic solution is globally stable, then $Q^{2}\left(h_{0}^{+}\right)<h_{0}^{+}$for any $h_{0}^{+} \in\left[\bar{h}, h_{T_{1}}\right]$. 
Theorem 4.2. In case $\left(C_{2}\right)$ (II), when $Q\left(h_{D_{1}}\right)<h_{D_{1}}$ and $Q^{2}\left(h_{0}^{+}\right)<h_{0}^{+}$for $\forall h_{0}^{+} \in\left(\bar{h}, h_{D_{1}}\right]$, the system(1) exists an order-1 periodic solution which is globally stable.

Proof. According to Theorem 3.2, we get that Poincaré map $Q(Z)$ exists a unique fixed point if $Q\left(h_{D_{1}}\right)<h_{D_{1}}$ and has no fixed point if $Q\left(h_{D_{2}}\right)>h_{D_{2}}$. So the order-1 periodic solution for system exists in $\left(0, h_{D_{1}}\right]$.

For $\forall h_{0}^{+} \in\left(\bar{h}, h_{D_{1}}\right]$, let $Q^{k}\left(h_{0}^{+}\right)=h_{k}^{+}$. According to $Q(Z)$ is monotonically decreasing on $\left(0, h_{D_{1}}\right]$, then we get $Q\left(h_{D_{1}}\right) \leq h_{1}^{+}<\bar{h}$. If $Q^{2}\left(h_{0}^{+}\right)<h_{0}^{+}$, then $\bar{h}<h_{2}^{+}<h_{0}^{+}<h_{D_{1}}$. By further deduction we get

$$
Q\left(h_{D_{1}}\right) \leq h_{1}^{+}<h_{3}^{+}<\ldots<h_{2 k+1}^{+}<\bar{h}<h_{2 k}^{+}<\ldots<h_{4}^{+}<h_{2}^{+}<h_{0}^{+}<h_{D_{1}} .
$$

Therefore we have $\lim _{k \rightarrow \infty} h_{2 k}^{+}=\lim _{k \rightarrow \infty} h_{2 k+1}^{+}=\bar{h}$. Then the order-1 periodic solution $Q(\bar{h})=\bar{h}$ is globally stable if $Q\left(h_{D_{1}}\right)<h_{D_{1}}$ and $Q^{2}\left(h_{0}^{+}\right)<h_{0}^{+}$for $\forall h_{0}^{+} \in\left(\bar{h}, h_{D_{1}}\right]$.

Theorem 4.3. In case $\left(C_{1}\right)$, if $Q\left(h_{T_{1}}\right)<h_{T_{1}}$ and $Q^{2}\left(h_{T_{1}}\right)<h_{T_{1}}$, then the stable order-1 or order-2 periodic solution exist for system (1).

Proof. For initial point $S_{0}^{+}\left(p_{0}^{+}, h_{0}^{+}\right)$in the phase set, where $p_{0}^{+}>0$ and $h_{0}^{+}>0$, we have a positive integer $k$ such that $h_{k}^{+}=Q^{k}\left(h_{0}^{+}\right)$holds after the pulse. When $h_{0}^{+}>h_{T_{1}}, Q(Z)$ is no fixed point on $\left[h_{T_{1}},+\infty\right)$ and monotonically increasing in this interval from Theorem 3.1. So there exists a integer $q$ such that $h_{q-1}^{+}>h_{T_{1}}$ and $h_{q}^{+}<h_{T_{1}}$. It follows that $h_{q}^{+}=Q\left(h_{q-1}^{+}\right)>Q\left(h_{T_{1}}\right)$, then $Q\left(h_{T_{1}}\right)<h_{q}^{+}<h_{T_{1}}$.

When $0<h_{0}^{+} \leq h_{T_{1}}, Q(Z)$ is monotonically decreasing in $\left(0, h_{T_{1}}\right]$ and $Q\left(h_{T_{1}}\right)<h_{T_{1}}$. Then we get $Q\left(h_{0}^{+}\right) \leq h_{T_{1}}$. From further analysis there exists an integer $q$ and $Q\left(h_{T_{1}}\right)<h_{q}^{+}<h_{T_{1}}$.

According to $Q(Z)$ is decreases monotonically and $Q^{2}(Z)$ is increases monotonically on $\left[Q\left(h_{T_{1}}\right), h_{T_{1}}\right]$, we get the following

$$
Q\left[Q\left(h_{T_{1}}\right), h_{T_{1}}\right]=\left[Q\left(h_{T_{1}}\right), Q^{2}\left(h_{T_{1}}\right)\right] \subset\left[Q\left(h_{T_{1}}\right), h_{T_{1}}\right]
$$

Therefore the periodic solution only needs to be studied in interval $\left[Q\left(h_{T_{1}}\right), h_{T_{1}}\right]$. Let $Q\left(h_{0}^{+}\right)=$ $h_{1}^{+} \neq h_{0}^{+}$and $Q^{2}\left(h_{0}^{+}\right)=h_{2}^{+} \neq h_{0}^{+}$, then system (1) has an order-1 or order-2 periodic solution. We analyze the four situations as follows:

(i) If $Q\left(h_{T_{1}}\right) \leq h_{2}^{+}<h_{0}^{+}<h_{1}^{+} \leq h_{T_{1}}$, then $Q\left(h_{T_{1}}\right) \leq Q^{2}\left(h_{0}^{+}\right)<h_{0}^{+}<Q\left(h_{0}^{+}\right) \leq h_{T_{1}}$.

From the monotonicity of $Q(Z)$ we obtain $h_{1}^{+}=Q\left(h_{0}^{+}\right)<Q\left(Q^{2}\left(h_{0}^{+}\right)\right)=Q^{3}\left(h_{0}^{+}\right)=h_{3}^{+}$, and $h_{4}^{+}=Q\left(Q^{3}\left(h_{0}^{+}\right)\right)<Q\left(Q\left(h_{0}^{+}\right)\right)=Q^{2}\left(h_{0}^{+}\right)=h_{2}^{+}$.

By mathematical induction, the relation is obtained as follows

$$
\begin{aligned}
Q\left(h_{T_{1}}\right) & \leq \ldots<Q^{2 k+2}\left(h_{0}^{+}\right)<Q^{2 k}\left(h_{0}^{+}\right)<\ldots<Q^{2}\left(h_{0}^{+}\right) \\
& <h_{0}^{+}<Q\left(h_{0}^{+}\right)<\ldots<Q^{2 k-1}\left(h_{0}^{+}\right)<Q^{2 k+1}\left(h_{0}^{+}\right) \leq h_{T_{1}} .
\end{aligned}
$$

(ii) If $Q\left(h_{T_{1}}\right) \leq h_{0}^{+}<h_{2}^{+}<h_{1}^{+} \leq h_{T_{1}}$, then $Q\left(h_{T_{1}}\right) \leq h_{0}^{+}<Q^{2}\left(h_{0}^{+}\right)<Q\left(h_{0}^{+}\right) \leq h_{T_{1}}$.

In this case, we get: $h_{3}^{+}=Q\left(Q^{2}\left(h_{0}^{+}\right)\right) Q^{3}\left(h_{0}^{+}\right)<Q\left(h_{0}^{+}\right)=h_{1}^{+}$, and $h_{2}^{+}=Q\left(Q\left(h_{0}^{+}\right)\right)=Q^{2}\left(h_{0}^{+}\right)<$ $Q\left(Q^{3}\left(h_{0}^{+}\right)\right)=h_{4}^{+}$. Furthermore, we get

$$
\begin{aligned}
Q\left(h_{T_{1}}\right) & \leq Q\left(h_{0}^{+}\right)<\ldots<Q^{2 k-1}\left(h_{0}^{+}\right)<Q^{2 k+1}\left(h_{0}^{+}\right)<\ldots \\
& <Q^{2 k+2}\left(h_{0}^{+}\right)<Q^{2 k}\left(h_{0}^{+}\right)<\ldots<Q^{2}\left(h_{0}^{+}\right)<h_{0}^{+}<h_{T_{1}} .
\end{aligned}
$$


(iii) If $Q\left(h_{T_{1}}\right) \leq h_{1}^{+}<h_{2}^{+}<h_{0}^{+} \leq h_{T_{1}}$. After the similar discussion we have

$$
\begin{aligned}
Q\left(h_{T_{1}}\right) & \leq Q\left(h_{0}^{+}\right)<\ldots<Q^{2 k-1}\left(h_{0}^{+}\right)<Q^{2 k+1}\left(h_{0}^{+}\right)<\ldots \\
& <Q^{2 k+2}\left(h_{0}^{+}\right)<Q^{2 k}\left(h_{0}^{+}\right)<\ldots<Q^{2}\left(h_{0}^{+}\right)<h_{0}^{+}<h_{T_{1}} .
\end{aligned}
$$

(iv) If $Q\left(h_{T_{1}}\right) \leq h_{1}^{+}<h_{0}^{+}<h_{2}^{+} \leq h_{T_{1}}$. Through the similar derivation we get

$$
\begin{aligned}
Q\left(h_{T_{1}}\right) & \leq \ldots<Q^{2 k+1}\left(h_{0}^{+}\right)<Q^{2 k-1}\left(h_{0}^{+}\right)<\ldots<Q\left(h_{0}^{+}\right) \\
& <h_{0}^{+}<Q^{2}\left(h_{0}^{+}\right)<\ldots<Q^{2 k}\left(h_{0}^{+}\right)<Q^{2 k+2}\left(h_{0}^{+}\right) \leq h_{T_{1}} .
\end{aligned}
$$

After analysis, the sequence $Q^{2 k}\left(h_{0}^{+}\right)$and $Q^{2 k+1}\left(h_{0}^{+}\right)$is bounded in situation (ii) and (iii). Thus, there exist values $\bar{h}$ such that $\lim _{k \rightarrow \infty} Q^{2 k}\left(h_{0}^{+}\right)=\lim _{k \rightarrow \infty} Q^{2 k+1}\left(h_{0}^{+}\right)=\bar{h}$ or exists different values $\bar{h}_{1}, \bar{h}_{2}$ such that $\lim _{k \rightarrow \infty} Q^{2 k}\left(h_{0}^{+}\right)=\bar{h}_{1}$ and $\lim _{k \rightarrow \infty} Q^{2 k+1}\left(h_{0}^{+}\right)=\bar{h}_{2}$, where $\bar{h}_{1} \neq \bar{h}_{2}$. In situation (i) and (iv), only the latter case holds. Therefore the system (1) has an order- 1 or order- 2 periodic solution.

Theorem 4.4. In case $\left(C_{1}\right)$, if $Q\left(h_{T_{1}}\right)=h_{T_{2}}^{+}<h_{T_{1}}$ and $Q^{2}\left(h_{T_{1}}\right)<h_{m_{1}}^{+}$, where $h_{m_{1}}^{+}=\min \left\{h^{+}, Q\left(h^{+}\right)=\right.$ $\left.h_{T_{1}}\right\}$, then the system has an order-3 periodic solution.

Proof. When $Q\left(h_{T_{1}}\right)=h_{T_{2}}^{+}<h_{T_{1}}$, the $Q(Z)$ has a unique fixed point $\bar{h} \in\left(Q\left(h_{T_{1}}\right), h_{T_{1}}\right)$. According to the continuity of $Q(Z)$ and $Q(\bar{h})=\bar{h}$, there exist $h_{m_{1}}^{+} \in(0, \bar{h})$ such that $Q\left(h_{m_{1}}^{+}\right)=h_{T_{1}}$. Let $G(h)=Q^{3}(h)-h$. Then, from the continuity of $G(h)$ and the expression of $Q(Z)$, we get

$$
Q^{3}\left(h_{m_{1}}^{+}\right)=Q^{2}\left(h_{T_{1}}\right)<h_{m_{1}}^{+} \text {and } Q^{3}(0)>0
$$

so $G\left(h_{m_{1}}^{+}\right)<0$ and $G(0)>0$.

Therefore there is $\tilde{h}$ in the interval $\left(0, h_{m_{1}}^{+}\right)$such that $Q^{3}(\tilde{h})=\tilde{h}$. Then the system $(1)$ has an order-3 periodic solution.

Remark 4.1. Same as the proof method of Theorem 4.4, we can get when $Q^{k-1}\left(h_{T_{1}}\right)<h_{m_{1}}^{+}$, the order- $k(k>2)$ periodic solution exist, and also the following Theorem 4.5.

Theorem 4.5. In case $\left(C_{2}\right)(I I)$, if $Q\left(h_{D_{1}}\right)=h_{A}^{+}<h_{D_{1}}$ and $Q^{2}\left(h_{D_{1}}\right)<h_{m_{2}}^{+}$, where $h_{m_{2}}^{+}=$ $\min \left\{h^{+}, F_{M}\left(h^{+}\right)=h_{D_{1}}\right\}$, then an order-3 periodic solution exist.

\section{Numerical simulations and discussion}

In this section, we numerically verify the theoretical results obtained in the previous section. To this end, we make $k=2.4, a=0.1, W=0.43, \eta=5, \mu=0.4, \tau=1.8, \sigma=0.8$.

When $u_{1}=1, v_{1}=0$ in the action threshold, the impulsive set and phase set are both straight lines, which is similar to most of the previous studies(Figure 4(a)). At this time, the threshold only related to the population density of the plankton. If we change the parameters in the action threshold as shown in figure 4(b) and (c), the impulsive set and phase set become two curves. And the degree of curvature of the curve changes with the change of $u_{1}$ and $v_{1}$. We get the population density and rate of change to determine the size of the action threshold, but the system (1) still has an order-1 periodic solution.

The change of the period $T$ under different thresholds is numerically analyzed as shown in Figure 5. We get that the control period under the fixed threshold is longer than the action threshold. And with the increase of parameter $a$, the period increases rapidly. When the action 

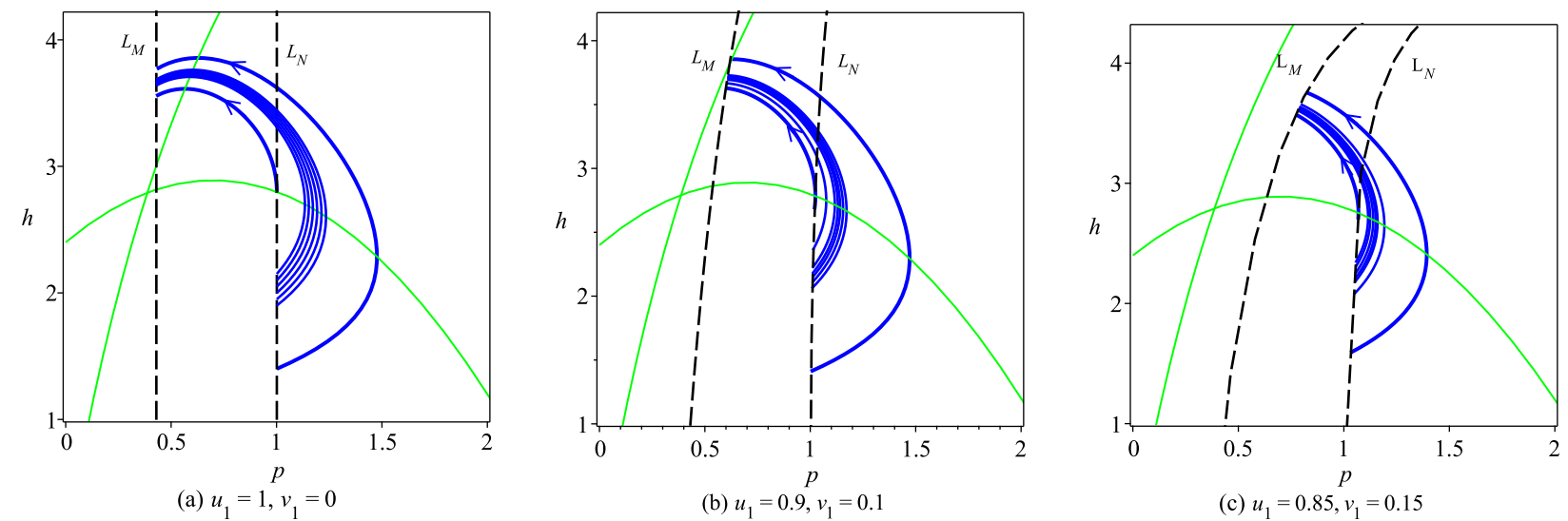

(c) $u_{1}=0.85, v_{1}=0.15$

Figure 4: The trajectories of system (1) under different action thresholds. The parameters fixed as $k=2.4, a=0.1, W=0.43, \eta=5, \tau=1.8, \mu=0.4, \sigma=0.8,(a) u_{1}=1, v_{1}=0,(b) u_{1}=0.9, v_{1}=$ 0.1 , and $(c) u_{1}=0.85, v_{1}=0.15$.

threshold is used, the control period decreases with parameter $v_{1}$ increases. As a result, herbivores can be caught in time so that people get more economic benefits.

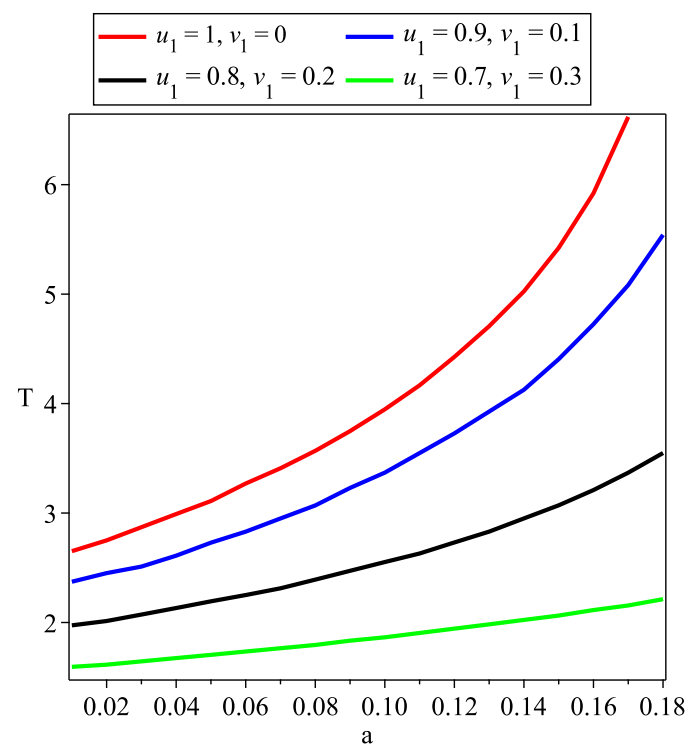

Figure 5: Effect of action threshold on period. The parameters fixed as $k=2, W=0.6, \eta=5, \tau=$ $1.8, \mu=0.4, \sigma=0.8$.

For case $\left(C_{1}\right)$, we set $k=2.4, a=0.1, W=0.43, \eta=5, \mu=0.4, \tau=1.8, \sigma=0.8, u_{1}=$ $0.9, v_{1}=0.1$. The system starts from the point $\left(p_{0}, h_{0}\right)=(1,0.25)$, such as Figure $6($ a) shown. Figure 6(b) and (c) respectively represent the time series of plankton and herbivore, respectively. Through the blue trajectory we get that the system has a stable order-1 periodic solution after the pulse. Here, the red trajectory indicates the solution of the system (1) without pulses. The comparison indicates that the density of the plankton and herbivore can be be maintained in a stable range under the state-dependent impulsive feedback control with action threshold. And when impulsive control is not adopted, the number of the plankton and herbivores rises in a short time and then drops to a small value. For case $\left(C_{2}\right)(\mathrm{II})$, the trajectory of the system $(1)$ starting 
from the point $\left(p_{0}, h_{0}\right)=(1.002,1.094)$ as shown in Figure 7. When $Q\left(h_{D_{1}}\right)<h_{D_{1}}$, we obtain that system (1) tends to the order-1 periodic solution (Figure 7(a)), and no order-1 periodic solution when $Q\left(h_{D_{2}}\right)>h_{D_{2}}($ Figure $7(\mathrm{~b}))$.
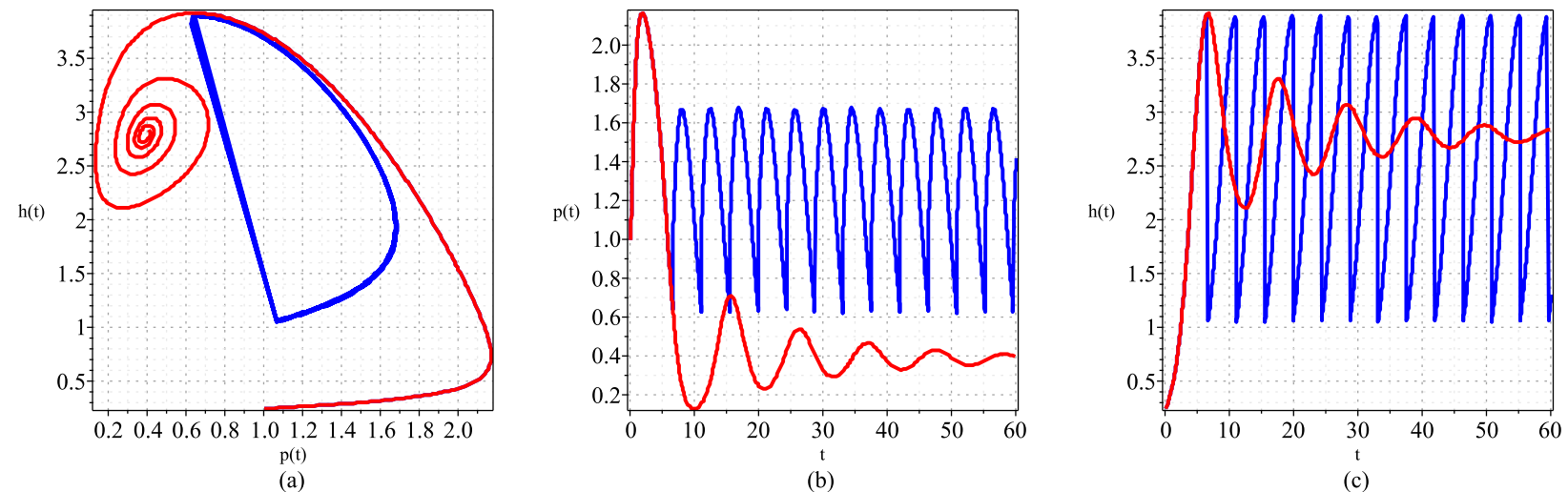

Figure 6: Phase portrait (a) and time series of plankton(b) and herbivore $(\mathrm{c})$ in case $\left(C_{1}\right)$. The parameters fixed as $k=2.4, a=0.1, W=0.43, \eta=5, \tau=1.8, \mu=0.4, \sigma=0.8, u_{1}=0.9, v_{1}=0.1$.

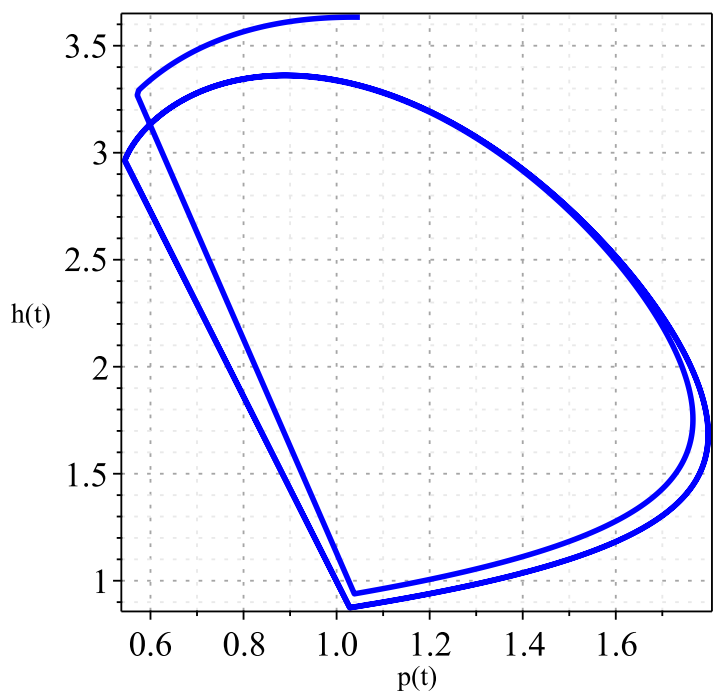

(a)

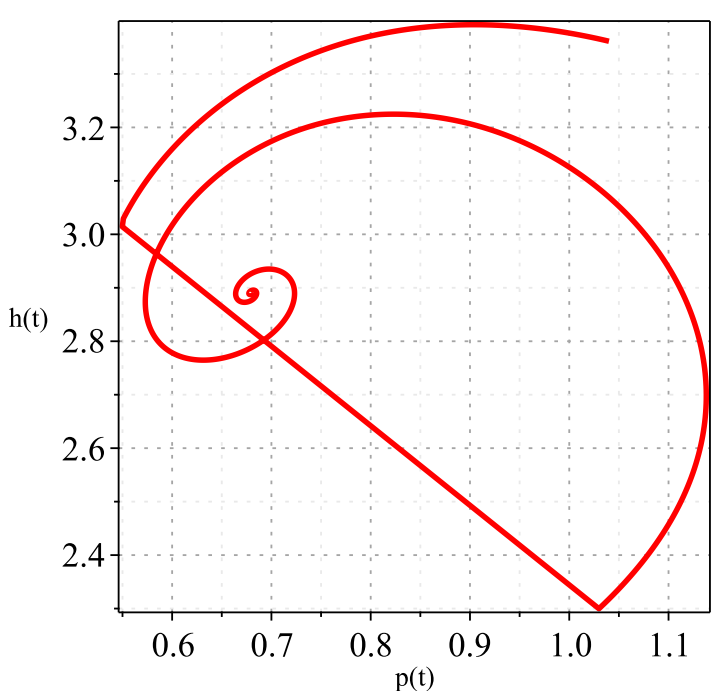

(b)

Figure 7: The trajectory of system $(1)$ in case $\left(C_{2}\right)(\mathrm{II})$. The parameters fixed as $k=2.4, a=$ $0.14, W=0.43, \eta=5, \tau=1.8, u_{1}=0.9, v_{1}=0.1(a) \mu=0.4, \sigma=0.8,(a) \mu=0.8, \sigma=0.3$.

The trajectories at different initial points in case $\left(C_{1}\right)$ and case $\left(C_{2}\right)(\mathrm{II})$ are shown in Figure 8. We can derive the trajectory starting from different points will tend to be the same order-1 periodic solution, which ultimately stabilizes. Therefore system (1) has the globally asymptotically stable order-1 periodic solution. 


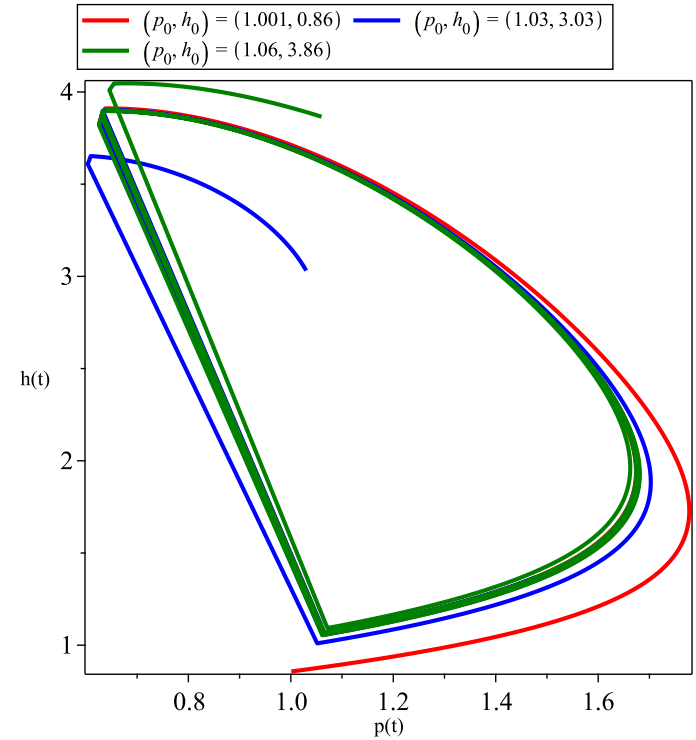

(a)

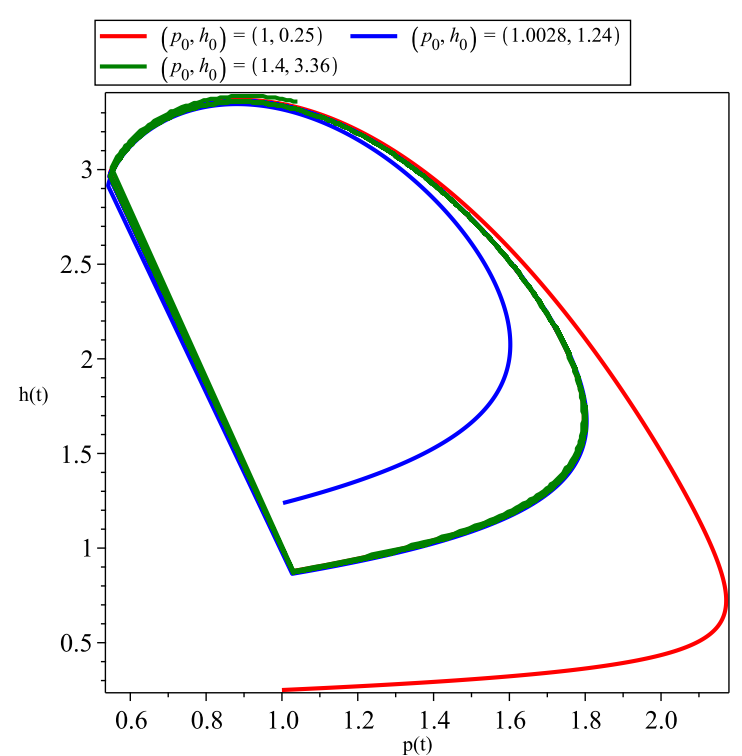

(b)

Figure 8: (a)The trajectory of system (1) from different initial points $(1.001,0.86),(1.03,3.03)$ and $(1.06,3.86)$ in case $\left(C_{1}\right)$. (b)The trajectory from different initial points $(1,0.25),(1.0028,1.24)$ and $(1.04,3.36)$ in case $\left(C_{2}\right)(\mathrm{II})$.

\section{Conclusion}

In this paper, we proposed a herbivore-plankton state-dependent impulsive model with action threshold and nonlinear impulsive function. We thoroughly studied the complex dynamics of the model and illustrated the influence of the action threshold on the system dynamics. The control form adopted in this paper is more in line with the development law of biological population, which makes herbivores harvest in time and obtain more economic benefits.

Compared with the existing work, for example [9], the innovation of this article is:

(1) The paper uses the action threshold determined by change rate and population density. The fixed threshold is a special case of the action threshold, and the action threshold is an extension of the fixed threshold. In this sense, our results generalise the results in [9]. Due to the integration of various factors affecting the population, the control method adopted in this paper is more general and the results obtained are more realistic.

(2) The change of control method makes the model have more complicated dynamic properties. The use of Poincaré map enables better understanding of the dynamics of the model.

(3) The dynamic characteristics of the model are studied more comprehensively and in details. We not only analyzed the order-1 periodic solution of the system, but also discussed the stability of the order- $\mathrm{k}(k \geq 2)$ periodic solution.

In the future research, in order to better analyze system dynamics, we will consider other impulsive population models with action thresholds. 


\section{Conflict of Interest:}

The authors declare that they have no conflict of interest.

Funding: This work is supported by the National Natural Science Foundation of China (11371230), the SDUST Research Fund (2014TDJH102), Shandong Provincial Natural Science Foundation, China (ZR2015AQ001), Joint Innovative Center for Safe And Effective Mining Technology and Equipment of Coal Resources, SDUST Innovation Fund for Graduate Students (SDKDYC170225). 


\section{Data availability statement}

The author declares that the data supporting the results of this study are available in the article. 


\section{References}

[1] B. Mukhopadhyay, R. Bhattacharyya, Role of gestation delay in a plankton-fish model under stochastic fluctuations, Mathematical Biosciences 215 (1) (2008) 26-34.

[2] Y. Lv, Y. Pei, S. Gao, C. Li, Harvesting of a phytoplankton-zooplankton model, Nonlinear Analysis Real World Applications 11 (5) (2010) 3608-3619.

[3] T. Zhang, X. Liu, X. Meng, T. Zhang, Spatio-temporal dynamics near the steady state of a planktonic system, Computers and Mathematics with Applications 75 (12) (2018) 4490-4504.

[4] X. Yu, S. Yuan, T. Zhang, Asymptotic properties of stochastic nutrient-plankton food chain models with nutrient recycling, Nonlinear Analysis Hybrid Systems 34 (2019) 209-225.

[5] X. Yu, S. Yuan, T. Zhang, Survival and ergodicity of a stochastic phytoplankton-zooplankton model with toxin-producing phytoplankton in an impulsive polluted environment, Applied Mathematics and Computation 347 (2019) 249-264.

[6] D. Jia, T. Zhang, S. Yuan, Pattern dynamics of a diffusive toxin producing phytoplanktonzooplankton model with three-dimensional patch, International Journal of Bifurcation and Chaos 29 (2019) Article Number: 1930011.

[7] S. Yan, D. Jia, T. Zhang, S. Yuan, Pattern dynamics in a diffusive predator-prey model with hunting cooperations, Chaos, Solitons and Fractals 130 (2020) Article Number: 109428.

[8] Y. Peng, Y. Li, T. Zhang, Global bifurcation in a toxin producing phytoplankton-zooplankton system with prey-taxis, Nonlinear Analysis: Real World Applications 61 (2021) Artical Number 103326.

[9] D. Fang, Y. Pei, Y. Lv, L. Chen, Periodicity induced by state feedback controls and driven by disparate dynamics of a herbivore-plankton model with cannibalism, Nonlinear Dynamics $90(5)(2017) 1-16$.

[10] S. Tang, B. Tang, A. Wang, Y. Xiao, Holling ii predator-prey impulsive semi-dynamic model with complex poincaré map, Nonlinear Dynamics 81 (3) (2015) 1575-1596.

[11] D. D. Bainov, P. S. Simeonov, Impulsive Differential Equation:Periodic Solutions and Applications, Pergamon Press, Inc, 2015.

[12] D. Li, H. Cheng, Y. Liu, Dynamic analysis of beddington-deangelis predator-prey system with nonlinear impulse feedback control, Complexity 2019.

[13] F. Wang, X. Zhang, Adaptive finite time control of nonlinear systems under time-varying actuator failures, IEEE Transactions on Systems Man and Cybernetics Systems (2018) 1-8.

[14] K. Ciesielski, On stability in impulsive dynamical systems, Bulletin of the Polish Academy of Sciences Mathematics 52 (84) (2010) 81-91.

[15] E. M. Bonotto, M. Federson, Limit sets and the poincare-bendixson theorem in impulsive semidynamical systems, Journal of Differential Equations 244 (9) (2008) 2334-2349. 
[16] Baek, Hunki, The dynamics of a predator-prey system with state-dependent feedback control, Abstract and Applied Analysis 2012 (2012) 1-17.

[17] J. Yang, S. Tang, Holling type ii predator-prey model with nonlinear pulse as state-dependent feedback control, Journal of Computational and Applied Mathematics 291 (2016) 225-241.

[18] H. Liu, H. Cheng, Dynamic analysis of a prey-predator model with state-dependent control strategy and square root response function, Advances in Difference Equations 2018 (1) (2018) 63.

[19] T. Li, W. Zhao, Periodic solution of a neutral delay leslie predator-prey model and the effect of random perturbation on the smith growth model, Complexity 2020 (2020) 15.

[20] Y. Li, Y. Li, Y. Liu, H. Cheng, Stability analysis and control optimization of a prey-predator model with linear feedback control, Discrete Dynamics in Nature and Society 2018 (2018) 12.

[21] Z. Shi, H. Cheng, Y. Liu, Y. Li, A cydia pomonella integrated management predator-prey model with smith growth and linear feedback control, IEEE Access 7 (1) (2019) 126066126076.

[22] Y. Wang, H. Cheng, Q. Li, Dynamic analysis of wild and sterile mosquito release model with poincaré map, Mathematical Biosciences and Engineering 6 (16) (2019) 7688-7706.

[23] Z. Shi, H. Cheng, Y. Wang, Optimization of an integrated feedback control for a pest management predator-prey model, Mathematical Biosciences and Engineering 16 (6) (2019) 79637981.

[24] C. Xu, S. Yuan, T. Zhang, Average break-even concentration in a simple chemostat model with telegraph noise, Nonlinear Analysis Hybrid Systems 29 (2018) 373-382.

[25] H. Qi, X. Leng, X. Meng, T. Zhang, Periodic solution and ergodic stationary distribution of seis dynamical systems with active and latent patients, Qualitative Theory of Dynamical Systems 18 (2) (2019) 347-369.

[26] T. Zhang, J. Wang, Y. Li, Z. Jiang, X. Han, Dynamics analysis of a delayed virus model with two different transmission methods and treatments, Advances in Difference Equations 2020 (1) (2020) 1.

[27] W. Wang, X. Lai, Global stability analysis of a viral infection model in a critical case, Mathematical Biosciences and Engineering 2020 (17) 1442-1449.

[28] D. Li, Y. Liu, H. Cheng, Dynamic complexity of a phytoplankton-fish model with the impulsive feedback control by means of poincaré map, Complexity 2020.

[29] Z. Jiang, W. Zhang, J. Zhang, T. Zhang, Dynamical analysis of a phytoplankton-zooplankton system with harvesting term and holling iii functional response, International Journal of Bifurcation and Chaos 28 (13) (2018) 1850162.

[30] Z. Zhong, L. Pang, X. Song, Optimal control of phytoplankton-fish model with the impulsive feedback control, Nonlinear Dynamics 88 (3) (2017) 2003-2011. 
[31] J. Yang, Y. Tan, Effects of pesticide dose on holling ii predator-prey model with feedback control, Journal of Biological Dynamics 12 (1) (2018) 527-550.

[32] Y. Wang, H. Cheng, Q. Li, Dynamical properties of a herbivore-plankton impulsive semidynamic system with eating behavior, Complexity 2020 (2020) 1-15. 